\title{
A novel missense mutation p.L76P in the GJB2 gene causing nonsyndromic recessive deafness in a Brazilian family
}

\author{
A.C. Batissoco ${ }^{1}$, M.T.B.M. Auricchio ${ }^{1}$, L. Kimura ${ }^{1}$, A. Tabith-Junior ${ }^{2}$ and \\ R.C. Mingroni-Netto ${ }^{1}$
}

${ }^{1}$ Centro de Estudos do Genoma Humano, Departamento de Genética e Biologia Evolutiva, Instituto de Biociências, Universidade de São Paulo, São Paulo, SP, Brasil

2Divisão de Educação e Reabilitação de Distúrbios da Comunicação, Pontifícia Universidade Católica, São Paulo, SP, Brasil

Correspondence to: R.C. Mingroni-Netto, Departamento de Genética e Biologia Evolutiva, USP, Caixa

Postal 11461, 05422-970 São Paulo, SP, Brasil

Fax: +55-11-3091-7478. E-mail: renetto@ib.usp.br

Mutations in the GJB2 gene, encoding connexin 26 (Cx26), are a major cause of nonsyndromic recessive hearing loss in many countries. We report here on a novel point mutation in GJB2, p.L76P (c.227C>T), in compound heterozygosity with a c.35delG mutation, in two Brazilian sibs, one presenting mild and the other profound nonsyndromic neurosensorial hearing impairment. Their father, who carried a wild-type allele and a p.L76P mutation, had normal hearing. The mutation leads to the substitution of leucine $(L)$ by proline $(P)$ at residue 76 , an evolutionarily conserved position in Cx26 as well as in other connexins. This mutation is predicted to affect the first extracellular domain (EC1) or the second transmembrane domain (TM2). EC1 is important for connexon-connexon interaction and for the control of channel voltage gating. The segregation of the c.227C>T (p.L76P) mutation together with c.35delG in this family indicates a recessive mode of inheritance. The association between the p.L76P mutation and hearing impairment is further supported by its absence in a normal hearing control group of 100 individuals, 50 European-Brazilians and 50 African-Brazilians.

Key words: GJB2 gene; Connexin 26; Hearing impairment; p.L76P; c.227C>T

Research supported by CEPID, FAPESP (\#1998/14254-2) and PRONEX-CNPq (\#140009/2007-8).

Received August 15, 2008. Accepted January 19, 2009

Hearing impairment is the most frequent and genetically heterogeneous neurosensory disorder. Epidemiologic studies have shown that hereditary nonsyndromic neurosensorial hearing impairment is predominantly inherited in an autosomal recessive manner (80\%), GJB2 being the most frequently mutated gene. This gene encodes the gap junction protein connexin 26 (Cx26) (1). With the exception of the GJB6 (connexin 30, Cx30) gene, connexin genes share the same architecture. A single large exon contains the entire coding region and is separated from its 5 ' untranslated region by an intron of variable size.

Most GJB2 mutations are located in the coding region of the gene (exon 2), with the exception of two splice site mutations (IVS $1+1 G>A$, IVS $1+3 G>A$ ) at the end of the noncoding exon 1 , and the mutation $-3438 \mathrm{C}>\mathrm{T}$ in the basal promoter region (2-4). To date, about 90 mutations of the GJB2 gene have been described, causing recessive hearing impairment (5). The c.35delG is particularly common in many populations, especially in the Mediterranean region (6), where it accounts for approximately $60 \%$ of GJB2 mutated alleles. In Brazil, it represents near $50 \%$ of the GJB2 mutations (7).

Connexins are constituents of connexons. Six connexins assemble into a connexon hemichannel, and the docking of two connexons from adjacent cells establishes gap junctions that constitute a major system of intercellular communication for the exchange of electro- 
lytes, second messengers and metabolites. Gap junctions seem to be essential for recycling potassium ions that are needed to initiate action potentials in hair cells (8). One postulated key role of these gap junctions in the sensory epithelia of the inner ear is in recycling potassium ions from the hair cells back to the endolymph in the auditory process (9). Immunochemical experiments have shown GJB2 expression in the stria vascularis, basement membrane, limbus, and the spiral prominence of the cochlea (10). A defective cochlear gap junction system can lead to hearing impairment.

We report here on a novel missense mutation, p.L76P (c.227C>T), in the coding region of the GJB2 gene associated with autosomal recessive nonsyndromic hearing loss. This mutation, which has not been previously described, was identified in two sibs with hearing impairment who carried the c.35delG mutation in trans.

The proband (Figure 1A, II-1) was a boy, born to nonconsanguineous clinically normal parents, and was referred to us at age of 12 years presenting hearing impairment. His 2-year-old sister also had hearing loss. The parents, both born in the city of São Paulo, were not affected by hearing impairment. No other cases of hearing impairment were reported in the family, and clinical evaluation of the children did not reveal symptoms or malformations that could suggest syndromic features. The following hearing measurements were performed in I-1, I-2, II-1 and II-2: acoustic immittance, including tympanometry and acoustic reflex thresholds, tonal and vocal audiometry with conditioning methods according to patients age. Pure tone audiometry was carried out to test

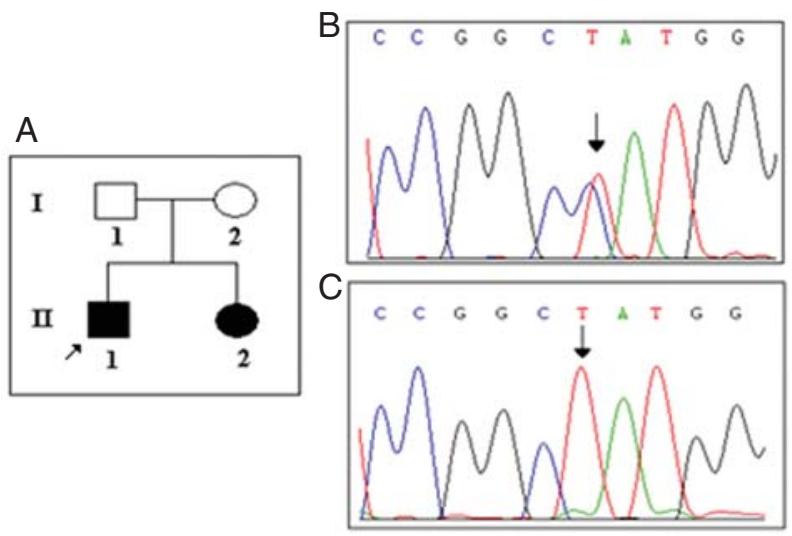

Figure 1. Novel mutation in the GJB2 gene. A, Pedigree of the family carrying the p.L76P (c.227C >T) mutation. The father (I-1) carries p.L76P in heterozygosis and the mother (I-2) carries c. 35 delG in heterozygosis. The sibs (II-1 and II-2) both carry p.L76P and c.35delG mutations. Sequence showing nucleotides corresponding to positions 222-231 of the GJB2 gene in the heterozygous father $(B)$ and wild-type sequence $(C)$. for air (250-8000 Hz) and bone conduction (250-4000 $\mathrm{Hz}$ ). Transiently evoked oto-acoustic emissions and distortion product were recorded for patients II-1 and II-2. A detailed clinical history was obtained and other causes of hearing impairment were excluded (neonatal complications, bacterial meningitis or other infections, use of ototoxic medication, and head trauma).

The study was approved by the Ethics Committee of Instituto de Biociências, Universidade de São Paulo, São Paulo, SP, Brazil, and written informed consent was obtained from all individuals or their legal guardians.

Genomic DNA was extracted from peripheral blood leukocytes by standard protocols. The proband and his sister were first screened for the c.35delG mutation in the GJB2 gene (11), and the $\operatorname{del}(G J B 6-D 13 S 1830)$ and del(GJB6-D13S1854) deletions in the GJB6 gene (12). The screening of the children revealed c.35delG in heterozygosis in both. Sequencing of the GJB2 coding region confirmed the c.35delG and indicated a $\mathrm{C}>\mathrm{T}$ substitution at nucleotide 227 (c.227C>T), leading to the replacement of a leucine by a proline at residue 76 (Figure $1 \mathrm{~B})$ in the 2 children. Their mother and father were found to be carriers of the c.35delG and the p.L76P mutation, respectively, and of normal alleles. The detected substitution has not been reported before (5, OMIM 121011).

In order to investigate the frequency of the novel c. $227 \mathrm{C}>\mathrm{T}$ in the general population, we screened a control sample consisting of 100 unrelated normal hearing individuals, 50 European-Brazilian and 50 African-Brazilians. Individuals who declared that the 4 grandparents were of European ancestry were considered to be European-Brazilians, and those who declared at least 1 grandparent of African ancestry were considered to be AfricanBrazilians. The analysis was performed with the MegaBace SNuPe genotyping kit (GE Healthcare, USA). None of the 100 controls carried the c.227C>T (p.L76P) mutation, thus decreasing the probability of it being polymorphic in the population.

The possible pathological nature of the substitution was pointed out by the analysis using PolyPhen (Polymorphism Phenotyping) (13,14), which indicated the c.227C>T (p.L76P) mutation to be possibly pathological.

The leucine amino acid at residue 76 of $\mathrm{Cx} 26$ is conserved among the different species (Figure 2) and among alpha and beta connexins (15), suggesting that this residue is crucial for the protein to be functional.

Connexins contain four transmembrane domains (TM1-TM4), two extracellular domains (EC1-EC2), one cytoplasmatic loop (CL), and $\mathrm{N}$ - and $\mathrm{C}$ - cytoplasmic termini (NT-CT). The N-terminal domain is involved in the process of membrane integration and hexamer forma- 
tion $(16,17)$ and, together with the first transmembrane domain, determines voltage gating. The extracellular loops regulate the connexon-connexon interactions, including heterotypic channel formation. Each loop contains three cysteine residues, conserved across all connexins and that form essential intramolecular disulfide bonds (18). The intracellular loop and C-terminal domains regulate $\mathrm{pH}$ gating (1). The TM domains are important for protein folding. The p.L76P mutation is predicted to affect the second transmembrane domain (TM2), but, according to some data bases, this position may be located at the highly conserved first extracellular loop (EC1) of Cx26, a region suggested to play a role in protein targeting. Other reported mutations that cause dominant ( $\mathrm{R} 75 \mathrm{~W}$ and $\mathrm{R} 75 \mathrm{Q}$ ) and recessive (W77X, W77R) hearing loss are located in the same region.

The patient described here is of predominantly European ancestry. Since the Brazilian population results from an admixture of three main geographic groups, Africans, Europeans and Native Americans, we cannot rule out a genetic contribution from Native Americans or Africans in this family. It is premature to speculate about the origin of the mutation. It is certainly a rare mutation. We have not detected it in a serial sample of 300 probands with hearing impairment, who had the complete coding region of the GJB2 gene investigated after SSCP analysis and sequencing (19). The screening of the c.35delG mutation in a total of 600 probands revealed 21 other heterozygotes (our unpublished data), and complete sequencing of the coding region of the GJB2 gene did not reveal the p.L76P

\section{References}

1. Bruzzone R, White TW, Paul DL. Connections with connexins: the molecular basis of direct intercellular signaling. Eur $J$ Bioch 1996; 15: 1-27.

2. Denoyelle F, Marlin S, Weil D, Moatti L, Chauvin P, Garabedian EN, et al. Clinical features of the prevalent form of childhood deafness, DFNB1, due to a connexin-26 gene defect: implications for genetic counselling. Lancet 1999; 353: 1298-1303.

3. Rabionet R, Gasparini P, Estivill X. Molecular genetics of hearing impairment due to mutations in gap junction genes encoding beta connexins. Hum Mutat 2000; 16: 190-202.

4. Matos TD, Caria H, Simoes-Teixeira H, Aasen T, Nickel R, Jagger DJ, et al. A novel hearing-loss-related mutation occurring in the GJB2 basal promoter. J Med Genet 2007; 44: 721-725.

5. Connexin deafness homepage. http://davinci.crg.es/deafness. Accessed June 24, 2008.

6. Denoyelle F, Weil D, Maw MA, Wilcox SA, Lench NJ, len-

\begin{tabular}{|c|c|}
\hline p.L76P & KN V C Y D H Y F P I S H I R P W A L \\
\hline Human & KN V C Y D H Y F P I S I R L W A L \\
\hline Rhesus & K N V C Y D H Y F P I S H I R L W A L \\
\hline Mouse & KN VCY D H HF P IS H I R L W A L \\
\hline Dog & KN V C Y D H Y F P I S H I R L A L \\
\hline Horse & KN VCY D H Y F P IS H I R L W A L \\
\hline Armadillo & KN V C Y D H Y F P I H I R L W A L \\
\hline Opossum & KN VCY D HF F P I S I R L W A L \\
\hline Platypus & KN VCY D H VF P IS H IR L W A L \\
\hline Lizard & KN V C Y D A F F P V S H R L W A L \\
\hline Chicken & RNVCY D HF F P I S I R L W A L \\
\hline$X_{-}$tropicalis & KN V C Y D H HF P V S H I L W C L \\
\hline Stickleback & KN VCY D H F F P S H IR L W C L \\
\hline
\end{tabular}

Figure 2. Alignment analysis of the p.L76P mutation in the connexin 26, GJB2 gene, in different species. Residue 76 is boxed.

mutation.

The hearing impairment was mild in the proband (I-1) and profound in his sister (II-2). The segregation of the c.227C>T (p.L76P) mutation together with c.35delG in this family indicates a recessive mode of inheritance, since the c.35delG heterozygous mother and the p.L76P heterozygous father both presented normal hearing.

Summing up, the presented family, population and bioinformatic data provide strong evidence for a causative association of the p.L76P mutation in the GJB2 gene with hearing impairment. The characterization of novel mutant alleles may contribute to a better understanding of the function of the connexin 26 domains.
Powell DR, et al. Prelingual deafness: high prevalence of a $30 \mathrm{delG}$ mutation in the connexin 26 gene. Hum Mol Genet 1997; 6: 2173-2177.

7. Christiani TV, Alexandrino F, de Oliveira CA, Amantini RC, Bevilacqua MC, Filho OA, et al. Molecular study in Brazilian cochlear implant recipients. Am J Med Genet A 2007; 143A: 1580-1582.

8. Holt JR, Corey DP. Ion channel defects in hereditary hearing loss. Neuron 1999; 22: 217-219.

9. Kikuchi T, Kimura RS, Paul DL, Takasaka T, Adams JC. Gap junction systems in the mammalian cochlea. Brain Res Brain Res Rev 2000; 32: 163-166.

10. Kikuchi T, Kimura RS, Paul DL, Adams JC. Gap junctions in the rat cochlea: immunohistochemical and ultrastructural analysis. Anat Embryol 1995; 191: 101-118.

11. Wilcox SA, Saunders K, Osborn AH, Arnold A, Wunderlich J, Kelly $T$, et al. High frequency hearing loss correlated with mutations in the GJB2 gene. Hum Genet 2000; 106: 399-405. 
12. del Castillo FJ, Rodriguez-Ballesteros $M$, Alvarez $A$, Hutchin $\mathrm{T}$, Leonardi $\mathrm{E}$, de Oliveira CA, et al. A novel deletion involving the connexin-30 gene, del(GJB6-d13s1854), found in trans with mutations in the GJB2 gene (connexin-26) in subjects with DFNB1 non-syndromic hearing impairment. $J$ Med Genet 2005; 42: 588-594.

13. Ramensky $V$, Bork $P$, Sunyaev $S$. Human non-synonymous SNPs: server and survey. Nucleic Acids Res 2002; 30: 3894-3900.

14. PolyPhen - polymorphism phenotyping. http://genetics.bwh. harvard.edu/pph. Accessed June 24, 2008.

15. USCS Genome bioinformatics. http://genome.ucsc.edu/. Accessed June 24, 2008.

16. D'Andrea P, Veronesi V, Bicego M, Melchionda S, Zelante
L, Di lorio E, et al. Hearing loss: frequency and functional studies of the most common connexin26 alleles. Biochem Biophys Res Commun 2002; 296: 685-691.

17. Zeilinger C, Steffens M, Kolb HA. Length of C-terminus of rCx46 influences oligomerization and hemichannel properties. Biochim Biophys Acta 2005; 1720: 35-43.

18. Dahl G, Werner R, Levine E, Rabadan-Diehl C. Mutational analysis of gap junction formation. Biophys $J$ 1992; 62: 172180.

19. Batissoco AC, Abreu-Silva RS, Braga MC, Lezirovitz K, DellaRosa V, Alfredo T Jr, et al. Prevalence of GJB2 (connexin26) and GJB6 (connexin-30) mutations in a cohort of 300 Brazilian hearing-impaired individuals: implications for diagnosis and genetic counseling. Ear Hear 2009; 30: 1-7. 